\title{
PHOTOGRAMMETRY DRIVEN TOOLS TO SUPPORT THE RESTORATION OF OPEN-AIR BRONZE SURFACES OF SCULPTURES: AN INTEGRATED SOLUTION STARTING FROM THE EXPERIENCE OF THE NEPTUNE FOUNTAIN IN BOLOGNA
}

\author{
F.I. Apollonio ${ }^{\text {a }}$, M. Gaiani ${ }^{\mathrm{a}, *}$, W. Basilissi ${ }^{\mathrm{b}}$, L. Rivaroli ${ }^{\mathrm{c}}$ \\ ${ }^{a}$ Dept. of Architecture - Alma Mater Studiorum University of Bologna, Italy - (fabrizio.apollonio, marco.gaiani)@unibo.it \\ ${ }^{\mathrm{b}}$ Istituto Superiore per la Conservazione ed il Restauro, Rome, Italy - vilma.basilissi@beniculturali.it \\ ${ }^{\mathrm{c}}$ Rome, Italy - laura_rivaroli@yahoo.it
}

Commission II

KEY WORDS: Photogrammetry, Conservation, 3D models, Colour management, Physically-based rendering, Bronze cleaning

\begin{abstract}
:
Checking the irreversible process of clean-up is a delicate task that requires a work of synthesis between theoretical knowledge and practical experience, to define an effective operating protocol on a limited patch area to be extended later to the entire artefact's surface. In this paper, we present a new, quick, semi-automated 3D photogrammetry-based solution to support restorers in the open-air bronze artwork cleaning from corrosion and weathering decay. The solution allows the conservators to assess in real time and with a high level of fidelity in colour and shape, the 'surfaces' to be cleaned before, during and after the clear-out treatment. The solution besides allows an effective and valuable support tool for restorers to identify the original layer of the bronze surface, developed and validated during the ongoing restoration of the Neptune Fountain in Bologna.
\end{abstract}

\section{INTRODUCTION}

Bronze is the result of an alloy between copper, tin and lead and is one of preferred material by the artists to make sculptural artefacts for both indoor and open air. Over time the action of the environment on the bronzes is expressed through the natural process of corrosion. The surface of a bronze object exposed to the air is where corrosion products are generated due to electrochemical corrosion and chemical reactions with atmospheric pollutants. These chemical products form different layers of corrosion, typically called 'patina', which offer partial protection to the metal substrate. The mechanisms of corrosion give to the metal a look different from the original, intentionally characterized by the artist's choice. The layer of an outdoor bronze patina consists mainly of copper oxides, copper sulphides and sulphates (green copper carbonates are extremely rare in atmospheric exposure). If healthy the layer is thin, stable and compact. If instead it is thick and dusty, it acts as wrap spongy and keeps corrosive agents and moisture in direct and continuous contact with the bronze surface. The presence of chlorides readily causes the localized formation of copper chlorides that is known to have a weakening effect on copper alloy patinas. The main problem in the bronze restoration is the removal of the unstable corrosion products, encrusted and laminated, on the metal surface, as much as those substances applied in previous restoration or maintenance (i.e. coatings layers, paints, etc.), while keeping intact the original patina. The cleaning and protection of the outdoors bronze artefacts is a difficult and complex task due to the corrosion mechanisms, that trigger the interaction between the material and the surrounding environment, and the difficult identification of the correct level of surface's cleaning. Therefore, the restorers, to identify what is the original surface of the artefact, should consider the stratification system that is distributed unevenly over the artwork (Basilissi \& Marabelli, 2008). The cleaning process is like a stratigraphic excavation: throughout different cleaning steps, they gradually advance, and analysing micro-areas they slowly proceed to identify the original surface layer. The evaluation and understanding of stratigraphy of the bronze's surface is currently tackled by a cautious and progressive operating procedure that is based primarily on the identification of representative sample areas indicative of the state of conservation; later the action is gradually taken with a sequence of cleaning test. When the supposed level is reached, the objective feedback is given by the chemical evaluation of the type of material removed - in relation to the selectivity of the applied products (solvents and reagents) - and with the surface's check using a magnifiers lenses and a digital microscope, able to ensure a microscale accuracy, but not a faithful colour reproduction. There are different analytical investigation techniques to characterize the deterioration of patina (Non-destructive Techniques or not), e.g. cross sections using reflected light microscopy and analytical techniques as infrared spectroscopy, Fourier-Transform Infrared Spectroscopy (FTIR) and FTIR microscopy in conjunction with Attenuated Total Reflection (ATR) spectroscopy, that makes also possible the surface mapping of the identified corrosion products (Mazzeo $\&$ Joseph, 2004), even if these are not quickly available to the restorer during the cleaning work. In any case to understand what is the proper layer we need not only experience of the restorer and specific tests, but also to cross heterogeneous data coming from different sources and fields of knowledge (restoration, chemistry, physics, art history...). To overcome this complex, and long procedure we developed a new, quick, semi-automated $3 \mathrm{D}$ photogrammetry-based solution able to allow the conservators to assess in real-time rendering (RTR) and with a high level of fidelity in colour and shape, the comparison of 'surfaces' before, during and after the cleaning treatment. The solution can be an effective and valuable support tool for restorers to reach and identify the original layer of the bronze surface, developed and validated during the ongoing restoration of the Neptune Fountain in Bologna. Bronze restorers are also authors of this paper and the procedure develop is conceived to

* Corresponding author 


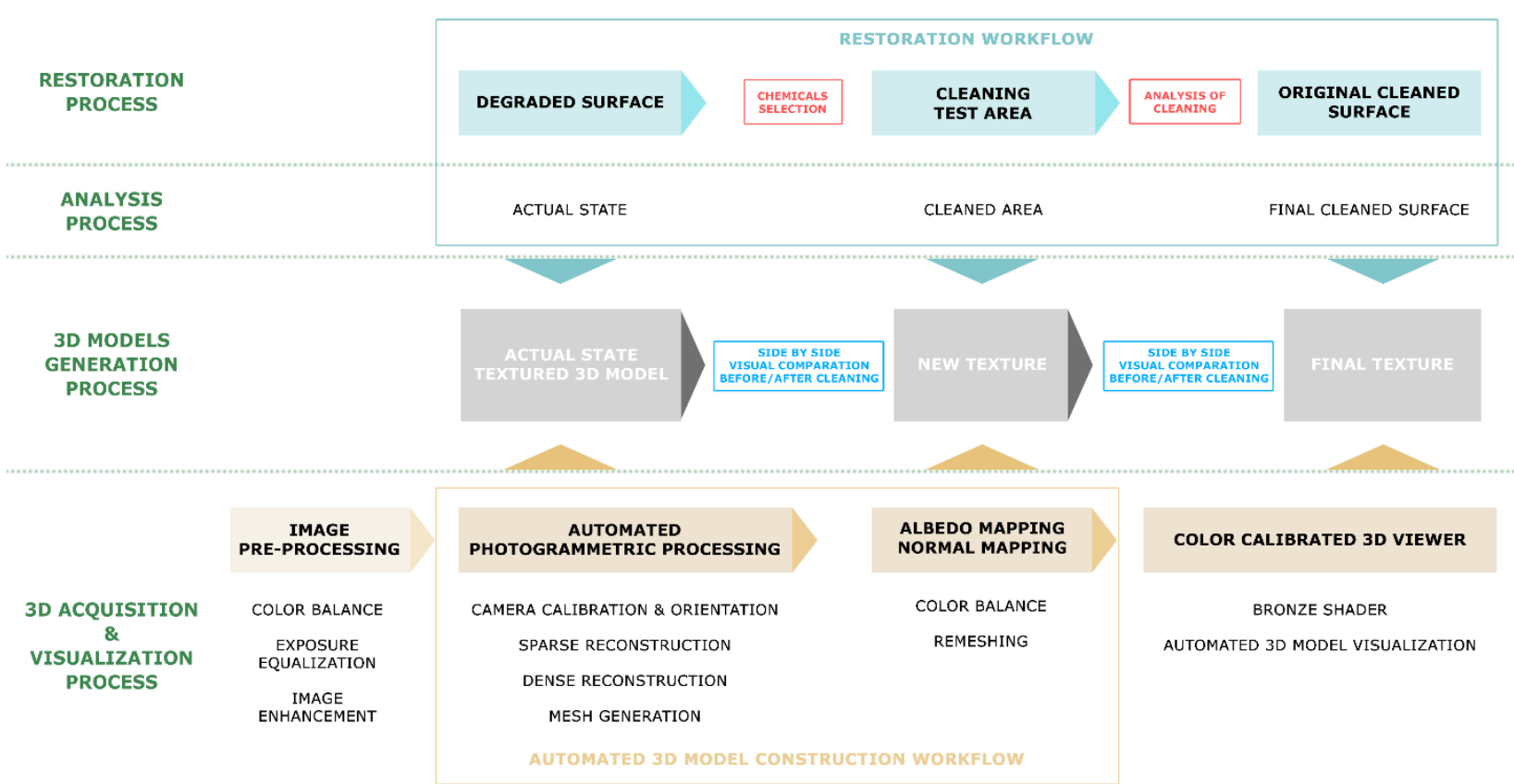

Figure 1. The proposed solution pipeline in parallel with the bronze cleaning phases.

be used by unskilled operators (i.e. the restorers). It aims not only to simplify and make more accurate the traditional procedure, but also to give a simpler and more accurate answer than the appearing solutions based on colorimetric measurements, using portable colorimeters or spectrophotometers (Franceschi et al., 2006; Luciano et al., 2006; Marchiafava et al., 2016).

\section{SOLUTION OVERVIEW}

Main problem faced in the paper is the correct visualization using OpenGL graphics of real material aging. Aging phenomena could be classified as a process that deteriorates objects over time. On metals, there are several external factors of deterioration generally gathered into two main classes: chemical and physical. Some effects of aging could lead to formation of corrosion products and introduce structural damage of the material, such as destructive corrosion that would destroy completely a metal surface over time (e.g. archaeological metal artefacts). A real-life surface might not be affected by just a single class of the ones but of any combination of different factors. Moreover, simultaneous processes could influence each other (Merillou \& Ghazanfarpour, 2008).

Various methods have been developed to simulate material aging, including patinas. A complete summary is given by Lu et al. (2007) and Rushmeier (2009). Nevertheless, results are limited and do not concern the faithful reproduction of real cases, that is our case. Starting from studies that demonstrated as openair bronze patinas presents average values between 80 and 130 $\mu \mathrm{m}$ were measured on the test areas before cleaning, which reduces to 50 to $110 \mu \mathrm{m}$ afterwards (Letardi, 2004) we modelled the material surface behaviour using the types of light interactions described in Westin et al. (1992), where they are roughly grouped by the size of the geometric structures and influenced by in three different levels:

- Macroscopic scale: features on this scale are low-frequency details can be regarded to define the shape and geometry of a model and will be stored as polygonal meshes;

- Mesoscopic scale: features on this scale are high-frequency details still just visible with the naked eye but are usually not considered to be part of the defining shape of an object (e.g., small bumps). Capturing these fine structures, using affordable methods, is the use of a data-driven representation that directly stores measured data in a form that can be used for a faithful depiction without being computationally too demanding: i.e. use of multiple textures, mapped onto the 3D geometry;

- Microscopic scale: on this level, the light is thought to interact with microscopically small facets on the surface of a material. The amount of reflected light can be influenced by the orientations of surface microfacets and the facets can occlude or cast shadows or inter-reflections on one another. A method usually adopted could model only the statistical distribution of the reflected light in dependence on the directions. In practice the key issue to solve is the use of the Bidirectional Reflectance Distribution Function (BRDF) (Nicodemus, 1965), that allows to model both the specular and diffuse reflectance components, as well as the bronze depressions and bumps.

The solution to the characterization of these three levels in the case of outdoor bronze surfaces has been defined as follows:

- Macroscopic scale: the shape of the surface has been reconstructed as a triangle mesh, with a $0.2 \mathrm{~mm}$ accuracy starting from a simple dataset of 9 images.

- Mesostructure scale: today's state of the art method for modelling the mesostructure is certainly the Bidirectional Texture Function (BTF), (Filip \& Haindl, 2009). To overtake the typical difficulties that characterized this method, a new solution starting from the observation of Kautz (2005), who show that in many cases acquiring some portions without making perceptible errors is enough. The new solution reconstructs the mesostructure, basically using a simple normal map obtained from a new procedure. Normal maps are an excellent solution to capture bronze surface mesostructure also because are easy to be stored in the modern graphics hardware (Akenine-Möller et al., 2008).

- Microstructure scale: to represent it, we developed a new shader able to overcome the typical problem of the completely Lambertian reflexion of the patinas and the presence of specular reflections in the case of the cleaned bronze using a unique BRDF representation. 

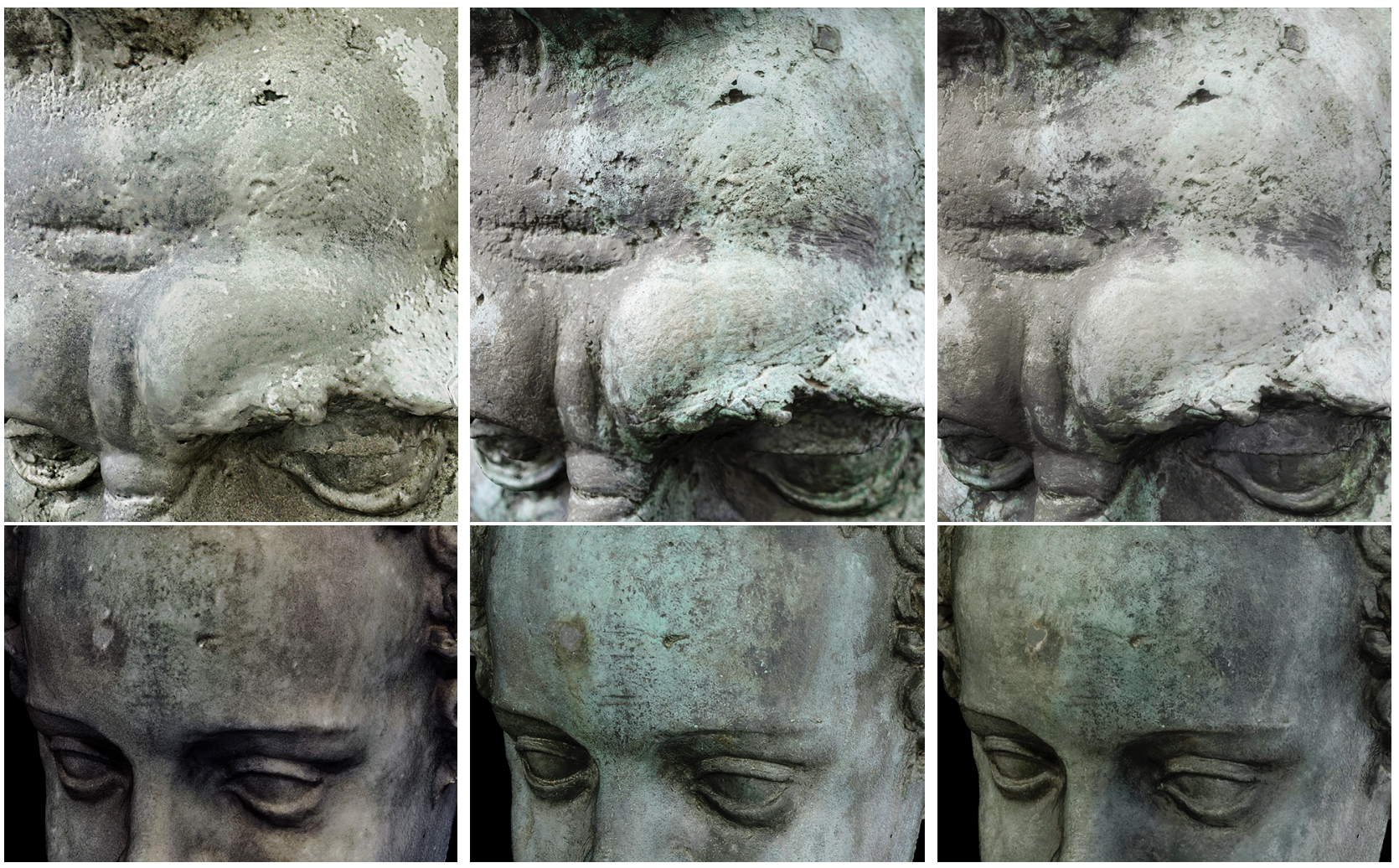

Figure 2. The result of our workflow: above the Neptune head and below the female Putto before cleaning and in two different cleaning phases, from left to right.

The proposed solution is depicted in Figure 1. Results are in Figure 2. From the point of view of the 3D model acquisition, construction and visualization it presents three distinct phases:

- The delivering of a photogrammetric reality-based 3D model with a $0.2 \mathrm{~mm}$ accuracy to model the macrostructure of the bronze surface, used as basis for all the cleaning phases;

- The acquisition and processing of some dataset of images to represent mesostructure and microstructure faithful modelling albedo and normal of the bronze surface;

- The side-by-side visualization of the textured 3D model display allowing the chromatic and microscale characteristics comparison in real time inside the OpenGL viewer through different phases of the surfaces cleaning.

The solution is based on our experience in the field of colour acquisition and reproduction, 3D photogrammetric pipeline automation (Gaiani, 2015) and real-time visualization (Gaiani et al., 2015), of Cultural Heritage artefacts.

From the point of view of the operator it requires:

- to place a target Xrite ColorChecker Classic in the scene;

- to take some images with a SLR camera, using a simple image sequence network geometry able to ensure correct camera calibration (Barazzetti et al., 2010) and orientation, and consistent data from dense stereo matching (Remondino et al., 2014) from automated process;

- to handle a button-click pipeline using a unique interface, specifying a measurement step to put in scale the 3D model.

The pipeline is a seven steps process:

1. Images pre-processing: develop the RAW image; denoise and colour balance

2. Automatic 3D model construction: align photo, build dense cloud, build mesh

3. 3D model scaling

4. 3D model remeshing and parametrization

5. Texture map building
6. Normal map building

7. 3D OpenGL based visualization.

Phases 1., 4., 5., 6., 7., are handled with in-house software, phases 2., 3., and partially 5. and 6. are managed using Agisoft Photoscan (Agisoft, 2017) and 7. is partially managed in the actual solution by Autodesk Showcase 2017 (Autodesk, 2017).

Today interface is based on a customized version of Photoscan, but in future is planned a web-based separate interface handling a new client server solution, as well as an in-house solution to replace Autodesk Showcase.

Automated image pre-processing move in the well-delineated context in which Stamatopoulos et al. (2012) demonstrated that a pre-processing approach for RAW imagery can yield significant photogrammetric accuracy improvements over those from JPEG images. We retained only the basic in-camera processing to avoid, as much as possible, modification of RAW pixel values. Pre-processing was done off-camera, using an automated procedure consisting in a calibrated and customized version of the on-camera processing, as in Ballabeni et al. (2015).

\section{PHOTOGRAMMETRIC DATA ACQUISITION}

The photogrammetric-based 3D model construction is based on the completely automated pre-processing procedure illustrated in (Gaiani et al., 2016a; Gaiani et al., 2016b), allowing a robust image matching starting from RAW photographs. The remaining phases of the automatic photogrammetric pipeline (Structurefrom-Motion, Bundle Adjustment, dense stereo matching), the mesh reconstruction and the texture mapping are based on the automation of Photoscan, with some customizations and enhancements. The software allows also a quick orientation of the new images captured in the different steps of the cleaning to obtain the new maps representing the albedo. 
We payed attention to appearing problems of automated tools to produce a metrically precise 3D point cloud or textured 3D model where apparently successful solution, are instead a local minimum and not the fully correct one. Typical examples are Structure-from-Motion results where, despite a message of successful image orientation and a very small re-projection error, there are some cameras wrongly oriented. A non-expert user could only spot such errors with difficultly and would proceed to the successive processing stages, negatively affecting the results. To minimize these problems in our automated solution, we:

- Established a minimal camera network, easy to use by the users, appropriate for each bronze surface shooting setting and assuming areas of maximum size of $30 \times 30 \times 30 \mathrm{~cm}$. The dataset includes 9 images vertical and horizontal two of these including a target Xrite ColorChecker Classic (CC) and acquired from a greater distance, the other seven acquired at the arc of a circle within an angle of $45^{\circ}$ from the perpendicular to the object;

- Automated all steps inside Photoscan to avoid human errors;

- Tested repeatedly all the step performed in Photoscan. Mainly we evaluated the cameras orientation using a different software solution consisting on our robust image matching based on a new implementation of the ASIFT detector-descriptor (Morel $\& \mathrm{Yu}, 2009)$ and a customized version of the software VisualSFM (Wu, 2013), slower but more accurate of the original software. We also tested the results made with the dense image matching against laser scanner data.

- Defined a very simple technique to put in scale the 3D model. The user need just to place markers in two different photos in correspondence of the four cross placed on corners of the CC. Distance between the cross are known, constant across different targets and accurately measured in Lab.

\section{COLOUR WORKFLOW}

Colour acquisition, management and visualization solution extends an existing efficient solution developed for an interior, light controlled environment (Gaiani et al., 2015), to the outside environment, with the limited working hypothesis of only indirect lighting always satisfied in the restoration yard of a statue. Implementation include:

1. colour management and calibration throughout the acquisition and rendering pipeline working in the context of colour space in which the images are observed (i.e., sRGB);

2. the development of a specific shader simulating the microstructure behaviour of the metallic material.

Mainly, we dealt with the issue of colour management for visualization using OpenGL API, an extremely limited system compared to the perceptive quality of the human eye and to that reproducible with analogical systems.

\subsection{Colour management and calibration}

The aim of our colour pre-processing consists essentially in obtaining radiometric calibrated albedo maps able to ensure the consistency and fidelity of surfaces colours reproduction at runtime in the visualization application OpenGL-based.

For digital images, colour characterization methods refer to the techniques of converting camera responses (e.g. RGB) to a device-independent colorimetric representation (e.g. CIEXYZ) (Green \& MacDonald, 2002). The main problem consists in recovering a linear relationship between the irradiance values and the pixel encoding produced by the camera, typically non-linear. We need therefore to model the non-linearities introduced by incamera processing for enhancing the visual quality of recorded images. Between the two general approaches to colour characterization - spectral sensitivities-based and colour targetsbased (Hong et al., 2001) - the latter technique is adopted, which uses a set of differently coloured samples measured with a spectrophotometer. The colour characterization is performed using a CC target (McCamy et al., 1976) in two different phases:

Captured images development and pre-processing

Albedo map pre-processing for rendering

The colour characterization in the second phase was introduced to overcome a limitation of Photoscan, which does not manage colour, producing unwanted artefact and - generally - colour differences even considerable due to mismatches between the colour spaces. The solution is a two-steps process.

In the first step, a first look-up table colour balance was performed against the $\mathrm{CC}$ using a 'fixed' approach realized with a known illuminant (e.g. the patches known values), i.e. using look-up tables (Viggiano, 2004). Practically is created an ICC profile, which was assigned, together with the rendered colour space, to the RAW image or to the processed albedo map. Before creating ICC profiles, we applied a standard gamma correction $(\gamma$ $=2.2$ ), converting all images to the camera's native linear colour space. This improve the profiles quality.

In the second step, a refinement is done using the ACR calibration scripts based on Fraser's calibration procedure for successive approximations (RAGS, 2017). Colour correction settings are applied automatically using a developed software solution, consisting in a RAW image processing allowing image demosaicing, white balance, output file in a rendered colour space, gamma correction, brightness control, 8-bit/16-bit conversion. Our automatic workflow is partially described in (Gaiani et al., 2016a) and is as follows:

1. RAW image 16-bit linearization and devignetting

2. ColorChecker localization

3. Polynomial fitting of observed values with expected values

4. Image correction using the fitting function found at point 3

5. White balance of the correct image

6. $\Delta \mathrm{E}^{*}{ }_{00}$ mean error evaluation on the $\mathrm{CC}$

7. Iterative image correction using the fitting function found at point 3 increasing the degree of the polynomial at each step; iteration stops when $\Delta \mathrm{E}^{*}{ }_{00}$ stops decreasing

8. Image correction using the new fitting function

9. ACR scripts calibration

10. Colour space assignment.

\subsection{Shader development}

To faithfully represent microstructure, reflectance can be described in terms of the BRDF that defines how light is reflected off an object's surface. Usually for metal and patinas simulations layered models are used, but this is not our case where:

- cleaned bronze surface present a slight specular reflection

- metallic patina result in a relatively rough and diffuse surface which completely covers the bronze before the cleaning

- cleaning operations could change specular reflection of surface in the different steps of cleaning.

Recent measurements on open-air bronze monuments (Franceschi et al., 2006) demonstrated that specular contribution is very limited and appears only when patinas cover a very limited area on the surface, that is at the end of cleaning.

Following these observations, we defined a BRDF incorporating a Lambert diffuse term and a microfacet BRDF for the specular contribution (Walter et al., 2007; Torrance \& Sparrow 1967; Cook \& Torrance, 1981):

$$
f(l, v)=\frac{c_{\text {diff }}}{\pi}+\frac{F(l, h) G 8 l, v, h) D(h)}{4(n \cdot l)(n \cdot v)}
$$

where $l$ light vector, $v$ view vector, $\boldsymbol{c}_{\text {diff }}$ diffuse albedo, $F(l, h)$ is the Fresnel reflectance, $G(l, v, h)$ is the proportion of microfacets 

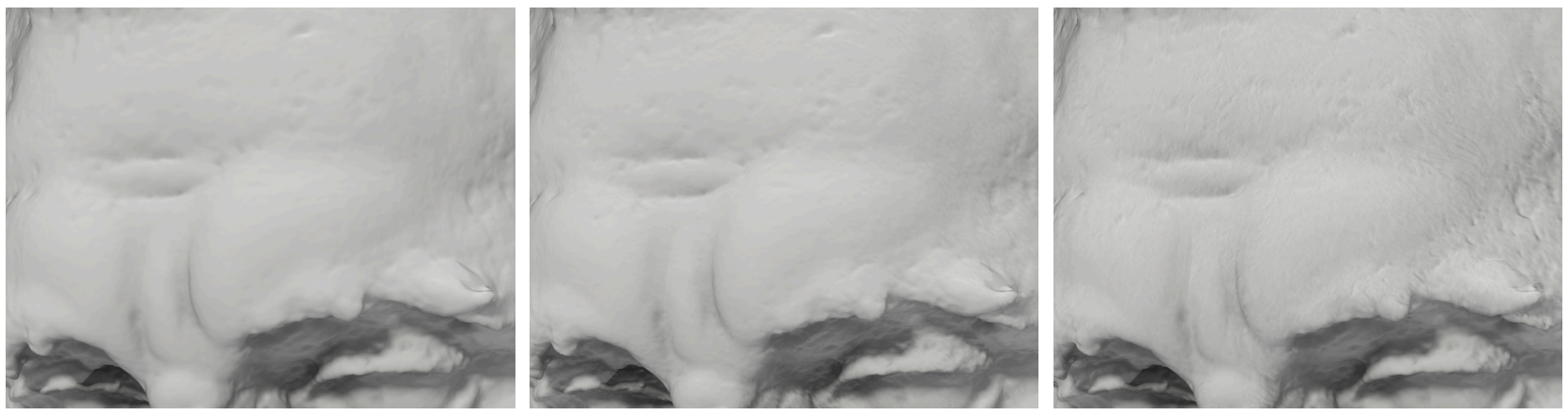

Figure 3. Normal mapping of the Neptune head, colour rendering: a. without normal mapping; b. using 3D modelling and baking from high-poly geometry technique; c. using our solution Sobel-filter based.

which are not shadowed or masked, $D(h)$ is the microfacet normal distribution function, determining the size, brightness, and shape of the specular highlight. A key step to define our $\mathrm{BRDF}$ is to have a Fresnel reflectance correct but practical to be use in RTR. Starting from Schlick's approximation (Schlick, 1994), we modelled Fresnel behaviour with two different values at $0^{\circ}$ and $90^{\circ}(0.8$ and 1.0$)$ and an interpolating curve.

We used the Fernando \& Kilgard (2003) solution with the parameters bias, scale, and power, as follows:

$$
F=\text { bias }+ \text { scale } *(1-h \cdot v)^{\text {power }}
$$

$G(l, v, h)$ in our case was fixed to 0.2 starting from direct observations. For $D(h)$ we used the Beckmann distribution which is physically-based and could deliver better results (Walter et al., 2007). In the Beckmann formula, the $m$ user-defined variable that controls the roughness of a surface, was placed to 0.5 as in Mihaylov (2013). Shader implementation was done using the OpenGL Shading Language (GLSL) where components are computed separately per-pixel and then added together to get the pixel's final colour.

\section{ALBEDO \& NORMAL MAPPING, MESH PROCESSING}

Our albedo and normal reconstruction is a two steps process:

- in the first step the selected set of images is projected on the mesh, exploiting found pose and camera calibration;

- in the second step a texture atlas is created.

Both these functions are well implemented in Photoscan reliably starting from Lempitsky \& Ivanov (2007) allowing excellent colour blending of images dataset and a compact atlas as output. We led enhancement in the parametrization to have less texture distortion introducing an extra-step consisting in the remesh of the geometry from dense stereo matching (typically noised and irregular) and exploited the excellent blending to develop a new solution for normal mapping.

The remeshing solution rely on the method described in Botsch $\&$ Kobbelt (2004), to create a curvature-adaptive isotropic mesh. The result is a new mesh that is curvature-adaptive, regular, and with less texture distortion. Maximal and minimal edge length of the output mesh and a maximal distance to the original mesh are predefined following typical features of bronze sculptures (edge length $0.2-1 \mathrm{~mm}$; max distance $0.1 \mathrm{~mm}$ ).

The normal mapping solution arise from the observation that we don't need a lower density surface to include finer detail, but accurately show these details. There are basically three methods to create normal maps. These methods can also be used together:

- 3D modelling and baking from high-poly geometry to a lowpoly geometry (Cohen et al., 1998; Cignoni et al., 1998);
- photometric stereo techniques (Woodham, 1980);

- 2D image processing, using filtering techniques over a heightmap, e.g. the Sobel filter (Pratt, 2007), which gives a greater weight to the directly adjacent neighbours (Ginsburg et al., 2001). In practice, the Sobel operator is simply a pair of $3 \times 3$ (separable) convolution kernels. One highlights the horizontal gradient/edges, and the other one highlights the vertical gradient/edges. The normal map could be obtained from the gradient vectors, normalized and displayed as an RGB-encoded normal map.

In our case the first two techniques are impractical and/or ineffective. Preserving attribute values on simplified meshes present the only advantage of a lighter polygonal model, but the quality of the mesostructure is that explicitly modelled. Shapefrom-shading is an ill-posed problem and constraints needed to give solution in the outside environment without allowing high accuracy detail. Normal maps created in 2D could be an excellent solution. Sobel operator is a simple way to approximate the gradient of the intensity in an image. However, it works best when tiled across 3D models that have a uniform direction in tangent space, like terrains or walls. On these models the UVs are not rotated; they are all facing roughly in the same direction. To get seamless lighting, rotated UVs require specific gradients in the normal map, which can only be created properly by baking a 3D model.

To fill this requirement, we developed a reconstruction pipeline that combines data from two different sources: the cameras orientations obtained from automated photogrammetry and a few 2D normal maps calculated using the Sobel operator starting from our dataset of 9 images. The solution is inspired, with some customization, by the work of Nehab et al. (2005), which proposed combining the low-frequency components of the geometry with the high-frequency details of the normal maps. Our pipeline consists of three main steps:

- Extraction of 2D normal maps applying the Sobel filter to the images used for the photogrammetric process converted on grey scaled diffuse image;

- Alignment of the normal maps to the mesh using existing camera orientation;

- Blending of multiple normal maps to produce a seamless normal field using Lempitsky \& Ivanov (2007).

The system is completely integrated in the photogrammetric pipeline and not requires further alignment, tedious preprocessing, or resolution/precision compatibility between different data types. Results are in Figure 3.

\section{DATA VISUALIZATION}

The developed 3D model visualization solution is based, at current state, on the RTR commercial software Showcase 2017, 
enabled by the Photoscan interface. It consists basically of an OpenGL user-friendly visualizer allowing a quick prototyping of the viewer and the automatic variants change of the different maps representing the colour of the bronze surface without any intervention of the user. The viewer supports texture maps of up to $16384 \times 16384$ pixels (needed to have the required visualization quality) requiring a midrange graphics card.

To improve visualization quality, four solutions have been implemented on the top of our RTR system:

1. Colour management: performed throughout the rendering pipeline by working within the context of the final colour space, in which images can be viewed (i.e., sRGB);

2. Shading enhancement: a pre-computed ambient occlusion and a series of light maps were implemented;

3. Colour balance by implementing the Ward \& EydelbergVileshin (2002) 'Picture Perfect RGB Rendering' technique in the sRGB version;

4. Image-based lighting (IBL) (Debevec, 2002) using as latitude-longitude HDR map a physically accurate model of the lighting conditions in the typical bronze surface observation scenario, synthetically recreated.

The main advantage of the system is a faithful visualization able to provide the ability to visualize the fine details of the surface at any angle of observation and under different types of lights. Restorers, as well as the scholars, can zoom in on high-resolution images to measure the characteristics of an image and compare different cleaning states side-by-side with cameras for both sides are locked, allowing to observe not only the cleaning level of the surface but also many extraordinary details until now, invisible.

\section{CASE STUDY AND ASSESSMENT}

The implemented workflow was evaluated exploiting the ongoing restoration works of the Neptune Fountain in Bologna, undoubtedly the most emblematic monumental complex of the city, and one of most beautiful Renaissance fountain worldwide (Tuttle, 2015). The restoration promoted by the Municipality of Bologna has been carrying out in collaboration with the University of Bologna and the Istituto Superiore per la Conservazione ed il Restauro (ISCR). One author of this paper is the coordinator for the bronze restoration.

We selected two case study concerning bronze surfaces consisting in an area of a female Putto and in an area of the head of the Neptune statue. Each case study includes three datasets, concerning distinct cleaning stages: pre, during, and at end of the restoration. The dataset (acquired with a Nikon D5300, CMOS Image sensor $23.5 \times 15.6 \mathrm{~mm}, 18-\mathrm{mm}$. nominal focal length) are of 9 images, two of which comprising the CC (Figures 5 and 6). We assessed both the case study in two different areas:

\begin{tabular}{|l|c|c|c|c|}
\hline \multicolumn{1}{|c|}{ File Name } & $\begin{array}{c}\Delta \mathrm{E}^{*}{ }_{00} \\
\text { Captured } \\
\text { images }\end{array}$ & $\begin{array}{c}\text { Exposure } \\
\text { error } \\
\text { Captured } \\
\text { images }\end{array}$ & $\begin{array}{c}\Delta \mathrm{E}^{*}{ }_{00} \\
\text { Albedo }\end{array}$ & $\begin{array}{c}\text { Exposure } \\
\text { error } \\
\text { Albedo }\end{array}$ \\
\hline Neptune before & 2.33 & -0.14 & 2.45 & -0.13 \\
\hline Neptune phase 1 & 2.80 & -0.10 & 2.71 & -0.08 \\
\hline Neptune phase 2 & 2.53 & -0.12 & 2.61 & -0.08 \\
\hline Putto before & 2.42 & 0.13 & 2.51 & -0.05 \\
\hline Putto phase 1 & 2.56 & 0.12 & 2.64 & -0.08 \\
\hline Putto phase 2 & 2.75 & 0.15 & 2.95 & -0.04 \\
\hline
\end{tabular}

Table 4. $\Delta \mathrm{E}^{*}{ }_{00}$ and exposure error (in f-stops) evaluation.
CIExyz chromaticity diagram (Sharma et al., 2005), and using the Imatest Studio software (Imatest, 2017). As reference, we used the 8-bit sRGB values tabulated by Pascale (2006). For all cases of study colour accuracy $\Delta \mathrm{E}^{*}{ }_{00}$ was $<3.0$. Applying the ACR scripts $\Delta \mathrm{E}^{*}{ }_{00}$ improved up to $<2.3$ both at the end of captured images development and pre-processing and of albedo map pre-processing for rendering. Exposure error in f-stops obtained were less than 0.05 f-stops. Results are in Table 4.

Photogrammetric pipeline: the implemented procedure was evaluated on the 9 image networks and the performances are reported using the statistical output of the bundle adjustment (reprojection error), camera location and accuracy evaluation of the dense matching results. To report camera alignment performances all datasets are oriented with different softwares (beside Photoscan also our customized version of Visual SFM). The accuracy evaluation of the polygonal model reconstruction results was done using Terrestrial Laser Scanning (Artec Space Spider, high-resolution 3D scanner based on blue light technology with nominal 3D resolution up to $0.1 \mathrm{~mm}$; 3D point accuracy up to $0.05 \mathrm{~mm}$; 3D accuracy over distance, up to $0.03 \%$ over $100 \mathrm{~cm}$ ) data as reference. The two polygonal models obtained by TLS were compared with the photogrammetric one.

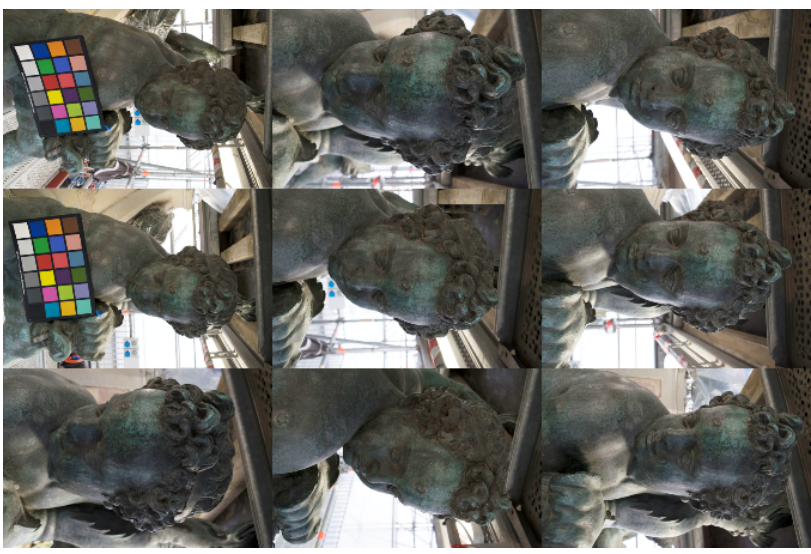

Figure 5. The female Putto datasets.

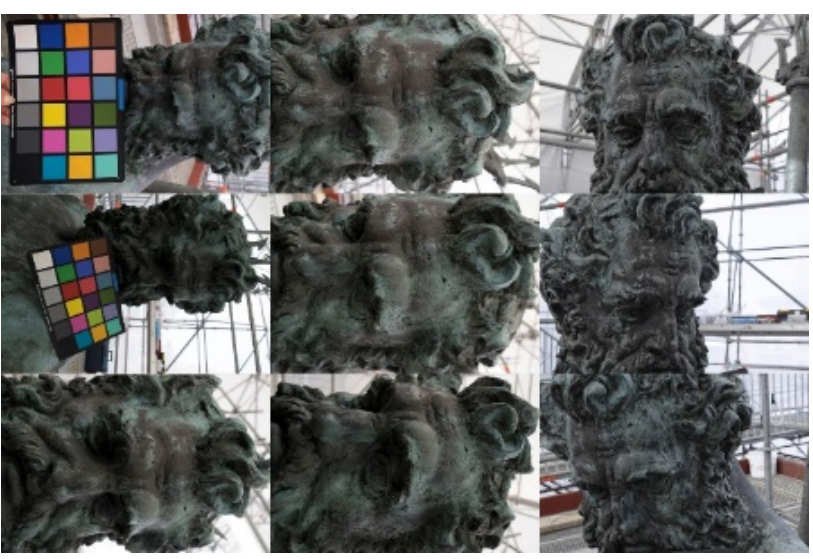

Figure 6. The Neptune head datasets.

The average image GSD (Ground Sample Distance) in both models (reference and data comparison) is ca. $1 \mathrm{~mm}$, therefore we had not to re-sampling any dense point clouds to have a reference comparable to the dense matching results. In Figures 7 and 8 the results show an excellent reliability and consistency.

Color fidelity: We assessed the colour accuracy of our characterization process using the metric distance $\Delta \mathrm{E}^{*}{ }_{00}$, on the 


\begin{tabular}{|l|l|l|l|}
\hline \multicolumn{2}{|c|}{ Female Putto datasets } & \multicolumn{2}{c|}{ Neptune head datasets } \\
\hline & & & \\
\end{tabular}

Figure 7. Image Based Polygonal modelling comparison with the ground truth TLS data; the histograms represent the point distribution errors of the mesh-to-mesh alignments: (r) Female Putto dataset; (1) Neptune head dataset.

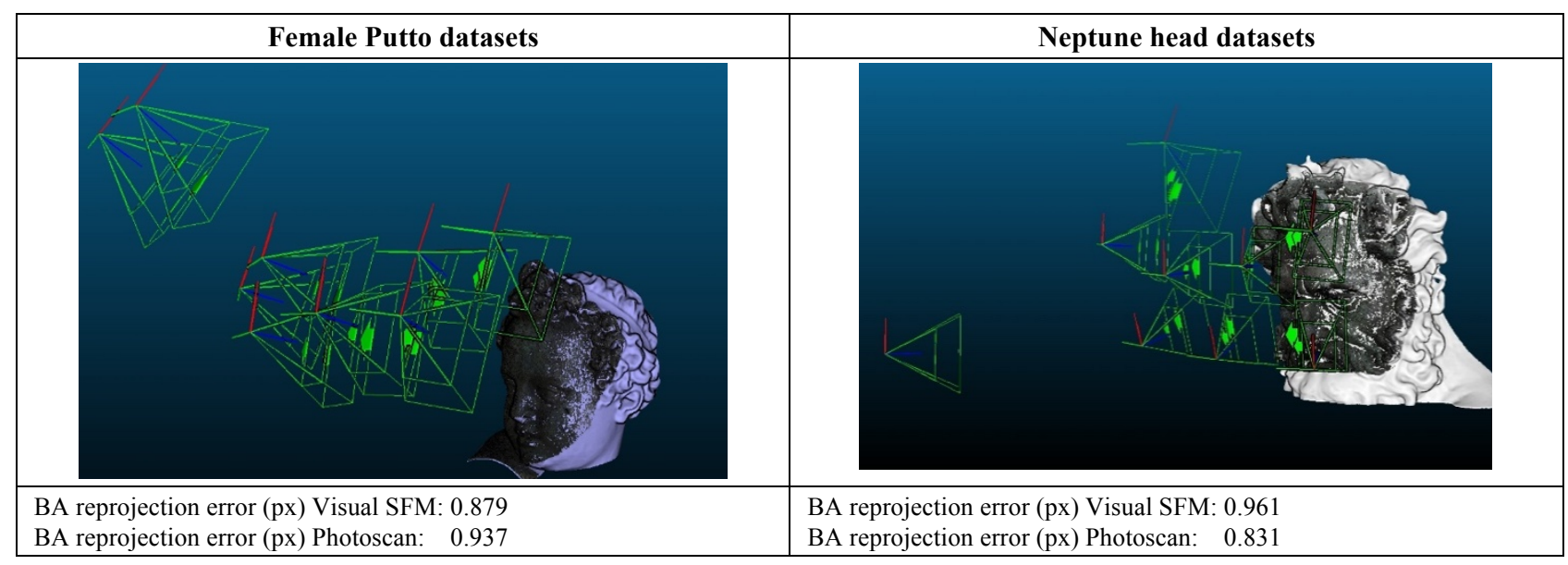

Figure 8. Camera alignment of female Putto (r) and Neptune head (1) datasets (9 images) comparison and Bundle Adjustment results.

\section{CONCLUSIONS AND FUTURE WORKS}

In this paper, we presented a global solution allowing to the restorer to have a useful control tool during the restoration work, especially for 3D artefacts. The software can be useful during cleaning phases for testing and gathering information (as direct feedback) of the reached results, considering the irreversible nature of the operation. Furthermore, it becomes an objective tool for documenting the restoration during the work and to illustrate it, giving an easy and direct access to other professionals interested. In addition, versatility of the system and its easy use it will be attractive and desirable to verify all its potentialities in lab, without the difficulties in the data acquisition phase (stability of the scaffolding, distribution of light, etc.) as showed during the experimentation carried out on a large bronze outdoors.

\section{ACKNOWLEDGEMENTS}

The authors are thankful to the Bologna City Council for their collaboration in the project. The authors acknowledge also M.C.M. srl for data from laser scanner.

\section{REFERENCES}

Agisoft, 2017. http://www.agisoft.com/

Akenine-Möller, T., Haines, E., Hoffman, N., 2008. Real-Time Rendering, Third Edition, A K Peters/CRC Press.
Apollonio, F., Ballabeni, A., Gaiani, M., Remondino, F., 2014. Evaluation of feature-based methods for automated network orientation. ISPRS Archives, XL(5), pp. 47-54.

Autodesk, 2017. http://www.autodesk.com/products/showcase/

Ballabeni, A., Apollonio, F. I., Gaiani, M., Remondino, F., 2015. Advances in image pre-processing to improve automated 3D reconstruction. ISPRS Archives, XL-5/W4, pp. 315-323.

Barazzetti, L., Scaioni, M., Remondino, F., 2010. Orientation and 3D modelling from markerless terrestrial images: combining accuracy with automation. The Photogrammetric Record, 25, pp. 356-381.

Basilissi, V., Marabelli, M., 2008. Le patine dei metalli: implicazioni teoriche, pratiche, conservative. In: Rinaldi S. (eds), L'arte fuori dal museo, Rome, pp. 74-89.

Botsch, M., Kobbelt L., 2004. A Remeshing Approach to Multiresolution Modeling. SGP '04 proceedings, ACM, New York, pp. 185-192.

Cignoni, P., Montani, C., Scopigno, R., Rocchini, C., 1998. A general method for preserving attribute values on simplified meshes. VIS ' 98 Proceedings IEEE Computer Society Press. Los Alamitos, pp. 59-66.

Cohen, J., Olano, M., Manocha, D., 1998. Appearance-preserving simplification. SIGGRAPH '98 Proceedings, ACM, NY, pp. 115-122.

Cook, R.L., Torrance, K.E., 1981. A reflectance model for computer graphics. Computer Graphics, 15(3), pp. 307-316. 
Debevec, P., 2002. Image-based lighting. IEEE Computer Graphics and Applications, 22(2), pp. 26-34.

Fernando, R., Kilgard, M.J., 2003. The Cg Tutorial: The Definitive Guide to Programmable Real-Time Graphics. Addison Wesley, Boston, MA.

Filip, J., Haindl, M., 2009. Bidirectional Texture Function Modeling: A State of the Art Survey, IEEE Trans. on PAMI, 31(11), pp. 1921-1940.

Franceschi, E., Letardi, P., Luciano, G., 2006. Colour measurements on patinas and coating system for outdoor bronze monuments. Journal of Cultural Heritage, 7(3), pp. 166-170.

Gaiani, M., 2015. I portici di Bologna. BUP, Bologna.

Gaiani, M., Apollonio, F.I., Clini, P., 2015. Innovative approach to the digital documentation and rendering of the total appearance of fine drawings and its validation on Leonardo's Vitruvian Man, Journal of Cultural Heritage. 16(6), pp. 805-812.

Gaiani, M., Apollonio, F. I., Ballabeni, A., Remondino, F., 2016a. A technique to ensure color fidelity in automatic photogrammetry. Colour and Colorimetry. Multidisciplinary Contributions, XII B, pp. 53-66.

Gaiani, M., Remondino, F., Apollonio, F. I., Ballabeni, A., 2016b. An Advanced Pre-Processing Pipeline to Improve Automated Photogrammetric Reconstructions of Architectural Scenes. Remote Sensing, 8(3), 178.

Ginsburg, D., Gosselin, D., 2001. Dynamic Per-Pixel Lighting Techniques. Game Programming Gems 2, pp 452-462.

Green, P., MacDonald, L.W., 2002. Colour Engineering: Achieving Device Independent Colour. John Wiley \& Sons, Hoboken, NJ.

Hong, G., Luo, M.R., Rhodes, P.A., 2001. A Study of Digital Camera Colorimetric Characterization Based on Polynomial Modeling. Color Research and Application, 26(1), pp. 76-84.

Horn, B. K. P., 1989. Obtaining shape from shading information. In: Shape from Shading. MIT Press Cambridge, Massachusetts, pp. 121-171.

IEC, 1999. Multimedia systems and equipment - Colour measurement and management - Part 2-1: Colour management - Default RGB colour space - sRGB. IEC 61966-2-1 (1999-10).

Imatest, 2017. http://www.imatest.com/products/imatest-master/

Kautz, J., 2005. Approximate Bidirectional Texture Functions. GPU Gems 2. Programming techniques for high-performance graphics and general-purpose computation. Addison-Wesley Professional, Boston.

Lempitsky, V., Ivanov, D., 2007. Seamless Mosaicing of Image-Based Texture Maps. IEEE CVPR proceedings, pp. 1-6.

Letardi, P., 2004. Laboratory and field tests on patinas and protective coating systems for outdoor bronze monuments. Metal 04 proceedings. National Museum of Australia, Canberra, pp. 379-387.

Lu, J., Georghiades, A.S., Glaser, A., Wu, H., Wei, L.Y., Guo, B., Dorsey, J., Rushmeier, H., 2007. Context-aware textures. ACM Trans. Graph., 26(1), Article 3.

Luciano, G., Leardi, R., Letardi, P., 2006. Principal component analysis of colour measurements of patinas and coating systems for outdoor bronze monuments. Journal of Cultural Heritage, 10(3), pp. 331-337.

Marchiafava, V., Cucci, C., Picollo, M., 2016. Colour measurement procedures on outdoor 3D artworks: a case study. Journal of the International Colour Association, 16, pp. 90-99.

Mazzeo, R., Joseph, E., 2004. Micro-destructive analytical investigation for conservation and restoration. Monumenti in bronzo all'aperto. Esperienze di conservazione a confronto, Nardini, Firenze, pp. 53-58.
McCamy, C.S., Marcus, H., Davidson, J.G.,1976. A Color Rendition Chart. Journal of Applied Photographic Engineering, 11(3), pp. 95-99.

Merillou, S., Ghazanfarpour, D., 2008. A survey of aging and weathering phenomena in computer graphic. Computers \& Graphics, 32(2), pp. 159174.

Mihaylov, V., 2013. Rendering Metals and Worn or Weathered Metallic Objects, Master's thesis, Technical University of Denmark.

Morel, J-M., Yu, G., 2009. ASIFT: a new framework for fully affine invariant comparison. SIAM Journal on Imaging Sciences, 2(2), pp. 438469.

Nehab, D., Rusinkiewicz, S., Davis, J., Ramamoorthi, R., 2005. Efficiently combining positions and normals for precise 3D geometry. ACM Trans. Graph., 24(3), pp. 536-543.

Nicodemus, F., 1965. Directional reflectance and emissivity of an opaque surface. Applied Optics, 4(7), pp. 767-775.

Pascale, D., 2006. RGB coordinates of the Macbeth ColorChecker. The BabelColor Company, Montreal.

Pratt, W. K., 2007. Digital Image Processing. PIKS Scientific Inside.

RAGS, 2017. http://www.rags-int-inc.com/PhotoTechStuff/

Remondino, F., Spera, M. G., Nocerino, E., Menna, F., Nex, F., 2014 State of the art in high density image matching. The Photogrammetric Record, 29, pp. 144-166.

Rushmeier, H., 2009. Computer Graphics Techniques for Capturing and Rendering the Appearance of Aging Materials. Service Life Prediction of Polymeric Materials, Springer, pp. 283-292.

Schlick, C., 1994. An Inexpensive BRDF Model for Physically-Based Rendering. Computer Graphics Forum, 13(3), pp. 233-246.

Sharma, G., Wu, W., Dalal, E.N., 2005. The CIEDE2000 ColorDifference Formula: Implementation Notes, Supplementary Test Data, and Mathematical Observations. Color Research and Application, 30(1) pp. 21-30.

Stamatopoulos, C., Fraser, C. S., Cronk, S., 2012. Accuracy aspects of utilizing raw imagery in photogrammetric measurement. ISPRS Annales, XXXIX-B5, pp. 387-392.

Torrance, K.E., Sparrow, E., 1967. Theory for off-specular reflection from roughened surfaces. J. Optical Soc. America, 57(9), pp. 1105-1114.

Tuttle, R.J., 2015. The Neptune fountain in Bologna. Bronze, marble and water in the making of a papal city. Brepols-Harvey Miller, New York.

Viggiano, J.A.S., 2004. Comparison of the accuracy of different white balancing options as quantified by their color constancy. Proceedings of the SPIE, 5301, SPIE Bellingham, WA, pp. 323-333.

Walter, B., Marschner, S.R., Li, H., Torrance, K.E., 2007. Microfacet models for refraction through rough surfaces. EGSR'07 Proceedings, The Eurographics Association, pp. 195-206.

Ward, G., Eydelberg-Vileshin, E., 2002. Picture perfect RGB rendering using spectral prefiltering and sharp color primaries. 13th EGWR Proceedings, ACM Press, New York, pp. 117-124.

Westin, S.H., Arvo, J., Torrance, K.E., 1992. Predicting reflectance functions from complex surfaces. SIGGRAPH '92 Proceedings, ACM, New York, pp. 255-264.

$\mathrm{Wu}, \mathrm{C} ., 2013$. Towards Linear-Time Incremental Structure from Motion, 3DV 2013 proceedings, pp.127-134. 